University of Nebraska - Lincoln

DigitalCommons@University of Nebraska - Lincoln

French Language and Literature Papers

Modern Languages and Literatures, Department

2021

\title{
Almuerz
}

David Lopez

Follow this and additional works at: https://digitalcommons.unl.edu/modlangfrench

Part of the French and Francophone Language and Literature Commons

Lopez, David, "Almuerz" (2021). French Language and Literature Papers. 73.

https://digitalcommons.unl.edu/modlangfrench/73

This Article is brought to you for free and open access by the Modern Languages and Literatures, Department of at DigitalCommons@University of Nebraska - Lincoln. It has been accepted for inclusion in French Language and Literature Papers by an authorized administrator of DigitalCommons@University of Nebraska - Lincoln. 


\section{David Lopez}

\section{Almuerz}

Elle m'a laissé le choix. Salsa amarilla o roja, avec les albondigues. J'ai dit amarille, abuela, c'est ce que je dis à chaque fois qu'elle me pose la question. Bueno elle a répondu, le sourire dans la voix, satisfaite à l'idée de satisfaire, et puis elle a ajouté que nunca elle s'accorde de ce que moi je préfère, parce que mi hermano también il a ça qu'il aime, et puis il vient plus souvent alors parfois elle se confond. Mais c'est vrai qu'on la laisse cociner, comme elle dit, et qu'elle connaît nos préférences, sachant qu'en général on aime bien quand ça se mange à la cuillère. Mais pour les albóndigas, l'exception n'a rien d'une gageure. Quand je lui demande si elle a besoin d'aide je sais déjà qu'au final je ne vais rien faire du tout, puisque je n'ai pas fini ma phrase que déjà elle me dit no no no, siéntate, si toi tú m'aides c'est cuando se termine, pa rebañar, et elle rit en retirant son tablier à toutes petites fleurs. D'accord, abuela.

Un rayon de soleil inonde la moitié de la table, que le store occulte à peine en cette position. D'ici on voit la montagne au loin, et je n'ai qu'à la regarder quelques secondes pour qu'elle me parle del abuelo, toujours assis là, sur la fin, à regarder cette montagne, en vanter les beautés et les avantages qu'il y a à rester là, assis sur cette chaise, à la contempler. Il

Published in Lydie Salvayre, maintenant même, ed. Warren Motte (Lincoln, NE : Zea Books, 2021). doi: 10.32873/unl.dc.zea.1287 
ne voulait plus vivir, me dit-elle comme si c'était la première fois. Quand son état s'est dégradé, le premier virus passé lui a quitté la vie. Mais c'est meilleur comme ça, elle ajoute en regardant dans le vide, et puis elle reprend sa contenance, pose sa main sur mon bras avec dépit, esta lista la comida, quedate à table yo arrive.

Je n'ai manifestement pas été convaincant quand j'ai dit que j'en avais assez dans l'assiette. C'est pas trop chaude? elle demande, en soufflant sur sa fourchette, et je dis esta bien, en soufflant aussi, pour l'imiter. À peine ça entre dans ma bouche que déjà je voyage dans le temps, cette saveur si familière de part son étrangeté, et puis je la regarde du coin de l'œil, avec le rictus en-dessous, je n'ai pas les mots pour exprimer le plaisir que je ressens alors on s'en tient à ce regard complice, qu'elle ponctue souvent d'un voilà, en s'apprêtant à attaquer l'assiette à son tour. Ça la met en joie comme ça n'a pas l'air non plus de la surprendre, et après quelques bouchées elle me pose la main sur le bras, et s'essuyant la bouche de l'autre main, elle me dit alors David, conta-moi, qu'est-ce qu'il t'arrive de nuevo, el libro qué tal, et je lui réponds que par-dessus tout j'aimerais avoir le temps d'écrire, que j'ai commencé quelque chose mais je navigue à vue, comme toujours. Pero tú trabajas verdad?, si abuela, bien seguro, comme on dit chez nous en fragnol. Ces derniers temps j'ai surtout rencontré mes lecteurs, ah bueno dit-elle avec enthousiasme, y esa gente ils disent quoi ? ils aiment ça que tú escrives ?, et je n'ai pas le temps de répondre qu'elle s'exclame que, de toute façon, ceux qui n'aiment pas c'est porqué ils ne connaissent rien de tout.

À la fin du repas elle me demande si je veux un dessert, et elle a beau savoir que je n'en mange jamais elle ne voudrait pas manquer la fois où, pour on ne sait quelle raison, j'en exprimerais le désir. Cafecito y ya, je dis, et la voilà partie vers 
la cuisine. Ces moments où elle s'absente pour s'affairer, autrefois, donnaient lieu à un tête à tête avec mon grand-père. Quand j'étais petit, c'était le moment propice à recevoir, pour moi, et dispenser pour lui, une leçon de vie. Souvent il me disait David, tú devois pas faire ça que tú devois pas faire, et j'ai mis du temps à comprendre qu'il me parlait de conformité à soi-même, de fidélité à ses principes, de tenir le cap qu'on s'est fixé. Ces dernières années, il semblait avoir renoncé à nous apprendre quoi que ce soit. Et ce tête-à-tête ne consistait plus qu'en une brève confession quant à sa lassitude envers la vie. Ces moments-là ne me manquent plus.

Alors qu'elle a déjà lavé les assiettes et tandis que le café coule, ma grand-mère revient s'installer à table, où je l'attends, dans un soupir. Elle approche sa chaise de la table ainsi que de moi dans le même geste, pose sa main sur mon bras. Bueno David, elle dit, et c'est quoi ça que tú escrives en ce moment alors? Je dodeline de la tête avant de répondre, elle sourit déjà, et je dis pas grand-chose abuela, pour l'instant j'ai peu de matière, je ne sais pas trop où ça va. Je lis, surtout. Ah bueno elle fait, et c'est quoi ça que tú lis aujourd'hui, et justement je saisis l'occasion pour lui parler de cette auteure, Lydie Salvayre, dont je me dis souvent qu'elle aimerait la lire.

Je lui raconte que la première fois que j'ai lu Lydie Salvayre j'ai été jaloux. Jaloux parce que le fragnol, d'abord. Depuis petit je connais ça le fragnol. On parle ça chez nous. On a notre propre lexique. On se foule le tobille, on a une écharde dans le ded, et la p'tite elle a des mocos. J'aurais voulu proposer ça, je dis à ma grand-mère, l'écrire en ne sachant pas qu'on l'a déjà fait avant, et ma grand-mère elle dit que c'est n'importa quoi ça que je dis, tú fais ça que tú veux, et je me rends à l'idée que pour ma grand-mère je n'ai qu'à faire quelque chose pour en être l'inventeur. 
Comme toujours j'ai demandé si je pouvais fumer et comme toujours elle m'a dit oui. Évoquer la guerre d'Espagne ça conduit systématiquement ma grand-mère à me parler de ces hommes qu'on libérait de prison et qu'on exécutait à la sortie, et de cet oncle, livré à ce sort, qui avait pu prendre un tram qui passait pile à ce moment-là. Elle est née pendant, ma grand-mère. Elle me raconte les souvenirs qu'on lui a laissés, et m'en laissera une partie. Je me suis déjà demandé si on m'avait tant parlé de cet oncle qui n'est pas mort à la seule fin de ne pas évoquer ceux qui le sont. Dans cet héritage qu'on me laisse, c'est de la vie qu'on me parle. C'est ce que je dis à ma grand-mère et j'ajoute tu vois abuela c'est ça, c'est ça qui m'a pris dans le livre, parce la meuf elle parle de la guerre avec sa mère et sa mère elle lui raconte quoi ?, elle lui raconte le plus bel été de sa vie !, la période où elle s'énamore, où elle est dans un genre de bouillonnement intellectuel, où elle s'émancipe. Parce que dans le livre, la guerre c'est pas le sujet, la guerre c'est le décor, un décor qui sert à faire ce que font les bons livres, raconter la vie. Sans artifice scénaristique ni posture idéologique. Et elle fait ça, elle, Lydie Salvayre, elle mentionne l'horreur, et puis elle te raconte la vie qui s'en accommode.

La fumée des cigarettes roulées accroche moins aux rideaux, pour ça qu'elle m'a laissé rester à table plutôt que m'approcher de la fenêtre. Je poursuis à propos de Salvayre, je dis que c'est ça qui m'intéresse justement, cette capacité à investir des sujets graves et d'en sacar la beauté, les traiter avec légèreté sans pour autant les vider de leur substance, sans que la menace ne s'éloigne, tapie tout près et prompte à changer le destin des personnages. Et tout ça sans pathos, mais comme ma grand-mère a un peu de mal avec ce mot je parle de misérabilisme, de cosa hecha para sacarte las lágrimas, de larmoyance donc, et j'ajoute que c'est un sacré 
numéro de funambule ce qu'elle propose, qu'elle est sans arrêt en équilibre sur une membrane, et qu'il s'agit d'avoir les chevilles souples. Ma grand-mère me dit pero quand même, c'est un sujet dur, como on s'impose une telle violence a sí mismo, toi aussi tú fais así David ?, et je ris, je réponds que oui, oui abuela, c'est une nécessité, qu'on le veuille ou non. Je lui cite le passage de Pas pleurer, très tôt dans le bouquin, où Salvayre parle du trouble que lui cause la jonction des récits de sa mère et de Bernanos, un trouble, dit-elle, dont elle craint qu'il ne l'entraîne là elle n'avait nullement l'envie d'aller. Car ce trouble implique d'envisager que les temps sombres n'empêchent pas la vie. Et que, pour instruit qu'on soit, on ne voit pas forcément venir. Ce trouble-là, c'est précisément où se situe le récit, dans ce va-et-vient constant entre atrocité et émerveillement. Et ça raconte quelque chose de l'endroit où se situe l'auteure, car je pense qu'on n'écrit jamais de choses aussi importantes que celles qu'on aurait préféré passer sous silence. C'est tout autant une tâche à accomplir qu'une responsabilité à assumer. Ainsi seulement le fardeau devient tremplin.

Le soleil a tourné un peu, c'est la table dans son intégralité qui est baignée de soleil maintenant. Ma grand-mère se lève pour baisser un peu le store, car j'ai le soleil dans les yeux. Quand elle se rassoie je reprends. Tu vois abuela, en fait Lydie Salvayre elle ne dit pas, elle montre, et ma grandmère se souvient m'avoir déjà entendu parler de ça. Je poursuis en insistant sur l'incarnation, oui abuela, encarnado sabes, ça permet de faire l'économie des discours, et puis ça caractérise de manière plus profonde et plus parlante les personnages. Après, c'est sûr que ça implique de montrer en deux pages ce qu'on pourrait dire en deux lignes. Mais ce qui est dit en deux lignes vit en moi le temps d'un chapitre, alors que ce qui m'est montré en deux pages vit en moi pour 
le temps du livre. Pour ça, ce qu'elle fait, c'est qu'elle utilise une écriture orale, où on entend les voix, les intonations, et d'un point de vue formel elle ne s'embarrasse même pas de la ponctuation, tu sais les guillemets et les tirets qu'on voit à chaque fois, non non, elle intègre la parole au récit. $\mathrm{Ma}$ grand-mère me fait remarquer que lorsque je parle de littérature mon débit s'accélère, alors j'essaie de m'adapter mais sur le coup je perds le fil, alors on rit ensemble. Siga, me interesa mucho lo que estas diciendo, me dit-elle. Alors j'en viens à dire que la grande force de Salvayre, à mon sens, c'est de toujours proposer un angle formel dans ses textes. Investir un sujet pour y raconter une histoire est une chose, mais se contenter de la chose racontée n'est pas suffisant. Chez Salvayre le quoi n'est pas une fin en soi, il est même une limite. Chez Salvayre, on sublime le quoi par le comment, ce qui implique que, peu importe ce qui est évoqué dans l'œuvre, c'est la littérature qui reste le sujet principal, et une réflexion sur celle-ci par la proposition esthétique. D'où le fait, d'ailleurs, que je perçois chez elle un rapport très ambivalent, duale, à l'écriture, car si son œuvre semble procéder d'une viscérale joie d'écrire elle paraît tout aussi réfractaire à la complaisance, en ce sens que cette joie d'écrire ne va pas de soi, elle se doit d'être conquise dans la confrontation à un sujet qui requiert, telle une condition sine qua non, d'y être impliqué émotionnellement. C'est comme partir d'un inconfort pour y créer son confort, là où le tourment parvient à rire de lui-même. C'est ce que je dis à ma grand-mère, mais pas vraiment comme ça.

J'avais justement Pas pleurer dans mon sac, car je le parcourais pour un travail qu'on m'avait proposé. Je lui mets dans les mains, elle le retourne et commence à lire la quatrième de couverture, à voix haute, parce qu'elle a toujours aimé ça, lire, à voix haute. Quand elle a terminé elle dit oui, 
ah oui je vais le lire, et puis, comme si elle venait de se souvenir de quelque chose, elle redresse la tête, me regarde, David, tú quieres una fruta o algo ?, et en riant je dis non, esta bien, puis bois une gorgée de café. Alors qu'elle ouvre le livre au hasard des pages, lisant des bribes de lignes à droite à gauche, je commence à lui parler de ce que je ressens de très espagnol dans cette littérature, et j'en reviens à évoquer le registre de langue, à cette oralité qui permet d'aller au plus près du parler des personnages, de leurs expressions, et de procéder à ce métissage linguistique si juste, si sonore, si ambivalent également, quand la langue littéraire rencontre le parler ordinaire, le langage de la rue, sans opposition, sans artifice non plus, simplement dans une grande maîtrise de ce que l'un peut apporter à l'autre. Ça donne quelque chose de sonore et de proprement jubilatoire. Quelque part, et alors qu'on pourrait y voir une manière de rejeter le classicisme en littérature, en vérité on le renforce, on le pérennise, en le nourrissant, en le renouvellant et l'enrichissant de tournures qui, plutôt que de s'y substituer, s'y greffent. Alors forcément, Lydie Salvayre ne fait pas l'économie de l'argot, ni même de la vulgarité, encore que chacun est libre d'appeler ça comme il le souhaite. Elle est d'accord avec moi, ma grand-mère, quand je dis que le mot « vulgarité » c'est pour les fragiles. Nous, on appelle ça la « familiarité », parce que la vulgarité on la voit davantage dans des attitudes, des idées, des postures, que des mots. Ce rapport désinhibé à la langue familière donc, franc et joyeux, capable d'inventions perpétuelles, suppose un rapport indéfectible à l'incarnation en littérature, car on ne peut faire l'économie du réel si l'on veut montrer, de manière authentique et véridique, un univers, et les gens qui le composent. Et ce rapport à la langue infuse bien au-delà de l'espagnol dans l'œuvre de Salvayre, qui toujours cherche à se placer à la frontière, qu'il s'agisse 
de celle qui sépare l'éthique de l'esthétique, ou bien celle entre le bon et le mauvais goût, entre l'oralité et l'écriture, ou bien encore celle qui prétendrait séparer la littérature du réel. Je bois la dernière gorgée de café, puis éteins ma cigarette dans le cendrier. Je parle du sens de l'équilibre, que je trouve admirable, chez Salvayre. L'usage de l'ironie, s'il peut être une pudeur, n'en reste pas moins une opportunité esthétique. C'est grâce à elle qu'elle arrive à jongler entre le grave et le léger sans jamais que ça jure. Ainsi l'impudeur n'a rien d'obscène. Parce que l'imperfection est acceptée d'emblée, dans le fond comme dans la forme, et on y reconnaît la vie, ambivalente et paradoxale, comme son écriture, perméable et poreuse, sujette à la circulation des registres, et s'érigeant presque contre toute conformité littéraire. Même la structure de ses livres ne prétend à aucune standardisation, au contraire, dans Pas pleurer on a plusieurs étages de narration et pourtant on s'affranchit de toute construction logique du récit, les histoires se mêlent et s'entrechoquent de la même manière qu'ont les souvenirs de surgir, cette manière foutraque comme un ricochet cérébral, qui implique que les enchaînements n'aient pas forcément de logique intellectuelle sinon émotionnelle, en ce sens que l'on progresse bien d'un sentiment, d'une sensation à l'autre, plutôt que d'un concept, d'une idée à l'autre, comme on le ferait dans un écrit explicatif, raisonné et ordonné, ce à quoi ne peuvent être sujets les récits de vie. La littérature de Salvayre n'est pas une représentation de la vie, mais bien la vie incarnée en texte, là où l'encre et le papier deviennent chair et os. Et je dis ça à ma grand-mère, en espérant qu'elle m'écoute encore, qu'elle comprendra où je veux finalement en venir à lui parler de tout ça, et je lui dis donc que pour moi la grande qualité de Lydie Salvayre c'est cette manière qu'elle a de ne pas écrire sur, de ne pas faire reposer la force 
de son texte sur son sujet mais bien dans une langue qui le servira, et donc d'écrire dans, d'écrire depuis, et qu'au final, mon sentiment dominant quand je lis ou bien évoque son écriture, c'est que moi aussi, j'ai envie d'écrire, que la lire me donne envie d'écrire, et ma grand-mère est satisfaite, quand elle entend ça.

On ne peut plus regarder la montagne en face sans être ébloui par le soleil mais on le fait quand même. Une autre cigarette, près de la fenêtre cette fois, pour sentir l'air. Elle va allumer l'écran, mettre le son très bas, et je vais m'allonger pour la sieste. Mais avant cela, je reste pensif quelques secondes, en regardant le siège de mon grand-père, tourné vers la montagne. 